\title{
Weight Loss Improves Cardio-Metabolic and Inflammatory State in Subjects with Metabolic Syndrome.
}

\author{
Matteo Della Porta ${ }^{1}$, Gabriele Piuri ${ }^{1}$, Micaela Garziano ${ }^{1,2}$, Michela Barichella ${ }^{3}$, Fulvio Muzio ${ }^{4}$ \\ Andy Joe Seelam ${ }^{5,6}$, Emma Assi ${ }^{5,7}$, Francesca D’Addio ${ }^{1,2}$, Paolo Fiorina ${ }^{1,2}$, Benvenuto Cestaro \\ and Roberta Cazzola' 1 \\ ${ }^{1}$ Dept. Biomedical and Clinical Sciences "L. Sacco", University of Milan, Milan, Italy, \\ ${ }^{2}$ International Center for T1D - Pediatric Clinical Research Center Romeo ed Enrica Invernizzi, Milan, Italy, \\ ${ }^{3}$ Clinical Nutrition Unit, Istituti Clinici di Perfezionamento, Milan, Italy, \\ ${ }^{4}$ Clinical Nutrition and Dietetic Unit,Luigi Sacco Hospital, Milan, Italy, \\ ${ }^{5}$ Dept. Biomedical and Clinical Sciences "L. Sacco", University of Milan, Milan, Italy, \\ ${ }^{6}$ International Center for T1D - Pediatric Clinical Research Center Romeo ed Enrica Invernizzi, Milan, Italy and \\ ${ }^{7}$ International Center for TID - Pediatric Clinical Research Center Romeo ed Enrica, Milan, Italy
}

\section{Abstract}

Metabolic syndrome (MetS) is a condition characterized by a constellation of reversible major risk factors for cardiovascular disease (CVD) and type 2 diabetes (T2DM). While it has been widely demonstrated that weight reduction by $5-10 \%$ decreases CVD and T2DM risk factors, including atherogenic dyslipidemia, on the other hand, its effects on comprehensive serum cytokine profile and endotoxemia are less investigated. Furthermore, the impact of weight loss on these parameters was studied especially in subjects with morbid obesity, often after bariatric surgery; while the studies on the effects of a physiological weight reduction with a balanced hypocaloric diet in overweight and moderately obese subjects showed contradictory results.

The aim of this pilot study was to investigate in overweight and obese men with MetS the effects of caloric restriction on the MetS-associated risk factors, chemical composition of lipoproteins and serum concentration of a wide spectrum of inflammation markers. In addition, the second purpose of this work was to study the possible correlation between lipoprotein chemical composition and these inflammation markers.

Eighteen adult Caucasian males $\left(25 \mathrm{~kg} / \mathrm{m}^{2}<\right.$ BMI $\left.<35 \mathrm{~kg} / \mathrm{m}^{2}\right)$ with MetS losing at least $5 \%$ of their initial weight after six months of a Mediterranean-style balanced hypo-caloric diet were included in the study. Lipoproteins were isolated from plasma by ultracentrifugation in a discontinuous $\mathrm{KBr}$ gradient. Lipoprotein concentrations of proteins, cholesterol, phospholipids, and triacylglycerols were determined by colorimetric assays. Peripheral cytokine levels (IL-2, IL-6, IL-7, IL-8, IL-10, IL-12, IL-17, GM-CSF, MCP-1, MIP-1b, IL1-b, IL-4, IL-5, IL-13, G-CSF, INF-g, TNF-a) were determined by Bioplex multiplex immunoassay. Endotoxemia was measured by Limulus Amebocyte lysate assay. The effects of weight-loss were analysed by Wilcoxon's test, the correlations between covariates by Spearman's test (significance: $\mathrm{p}$-value $\leq 0.05$ ).

After weight loss, we observed an improvement of MetS-associated risk factors and changes in lipoproteins composition. In particular, together with a reduction of triglyceridemia, we detected a massive transfer of triacylglycerols from HDLs toward LDLs. Furthermore, a significant decrease of IL- $6(0.9 \pm 0.7$ vs $0.5 \pm 0.6)$, TNF- $\alpha(0.7 \pm 0.3$ vs $0.3 \pm 0.18)$, IL-8 (1.6 \pm 0.7 vs $1.3 \pm 0.3)$ and MIP-1 $\beta(19.2 \pm 1.5$ vs $18.5 \pm 1.5)$ was observed. Finally, peripheral levels of TNF- $\alpha$ and IL-8 were directly correlated with all lipid species of VLDL, whereas the concentration of MIP-1 $\beta$ and endotoxin with HDL lipids.

Weight loss improved cardio-metabolic risk factors and decreased inflammatory state by reduction of cytokine levels. The relationships between lipoprotein composition and serum inflammation markers deserve to be deepened by studying broader populations.

\section{Conflict of Interest}

There is no conflict of interest 